\title{
Modeling the Transmission and Dynamics of COVID-19 Using Self-protection and Isolation as Control Measures
}

\author{
Demsis Dejene, Tesfaye Worku, Purnachandra Rao Koya \\ Department of Mathematics, Wollega University, Nekemte, Ethiopia \\ Email address: \\ demsdej@gmail.com (D. Dejene), tesfworku21@gmail.com (T. Worku),drkpraophd@gmail.com (P. R. Koya) \\ To cite this article: \\ Demsis Dejene, Tesfaye Worku, Purnachandra Rao Koya. Modeling the Transmission and Dynamics of COVID-19 Using Self-protection \\ and Isolation as Control Measures. Mathematical Modelling and Applications. Vol. 5, No. 3, 2020, pp. 191-201. \\ doi: 10.11648/j.mma.20200503.18
}

Received: June 8, 2020; Accepted: June 28, 2020; Published: September 16, 2020

\begin{abstract}
In this paper, a deterministic five compartmental mathematical model is developed and conducted simulations to study the dynamics of COVID-19 with the inclusion of self-protection and isolation as control measures. The model is shown mathematically and biologically valid by verifying that the solutions are both positive and bounded. Using next generation matrix method, the reproduction number is formulated. The disease free equilibrium point is found and shown that it is conditionally locally and globally asymptotically stable. Further, following Lyapunov function method Endemic equilibrium point is found and shown that it is conditionally globally asymptotically stable. Numerical simulation study is conducted by assigning reasonable values to the parameters. It is concluded that the spread of the disease can be brought under control if the control measures like Self-protection including social distancing and Isolation are implemented affectively. The results and the discussion are presented in the body of the paper lucidly.
\end{abstract}

Keywords: COVID-19, Self-Protection, Isolation, Equilibria, Stability, Numerical Simulations

\section{Introduction}

A Coronavirus Disease 2019 or COVID-19 is a respiratory illness that can spread from human to human through respiratory droplets. Some cases of the disease were firstly reported in Wuhan China in the winter month of December 2019. However, the intermediate host for COVID-19 couldn't have been so far investigated and identified. Also, it is not yet clear whether this novel virus infected humans either by direct transmission from bats or through intermediate hosts $[1,2]$.

Coronavirus is appearing and disturbing human kind in many other forms. A new coronavirus COVI-19 has been identified to cause respiratory illness which is atypically associated with the common cold and pneumonia. Middle East Respiratory Syndrome Coronavirus or MERS-COV and Serve Acute Respiratory Syndrome Coronavirus or SARSCOV are still other forms and are also found in humans [3]. SARS-CoV and MERS-CoV originated from bats. Both these viruses infect humans through different jumping species and intermediate hosts [4]. It is suspected that honor civets sold in live animal markets were the intermediate host for SARS-
$\mathrm{CoV}$ [5]. MERS-CoV was transmitted through dromedary camels [6-9].

In general, for these type of coronavirus diseases there are mainly two types of transmission routes: one is animal-tohuman transmission and the other is human-to-human transmission. The virus transmission occurs when infected and susceptible populations stay in close contact with one another. The virus transmits to a susceptible human through respiratory droplets produced when an infected person coughs or sneezes. It may also be possible that a person can get exposed to coronaviruses disease if the person touches a surface or object that has the virus on it and then touches person's own mouth, nose, or eyes [10-14].

Generally, COVID-19 is caused by the SARS-CoV-2 virus which belongs to the family of coronaviruses [15]. According to WHO the patients infected by COVID-19 will have symptoms including fever, cough and shortness of breath, and the severe patients may have renal failure. However, hospital based survey reveals that humans do not show any immediate clinical symptoms. It is examined that the outbreak of COVID-19 had an incubation period of 2 to 14 days i.e., during this period the infected humans are not infectious 
[16]. Furthermore, it is an asymptomatic sickness to pneumonia [17-19].

In laboratory tests it is found that the individuals having low defense cells and white blood cell are more vulnerable to corona virus [20, 21]. Corona virus infection develops in LLC-MK2 cells and also in Vero cells [22]. The virus infection results in more serious illness in individuals having weakened immune systems, older people, and those individuals with chronic diseases such as renal disease, diabetes, cancer, and lung diseases.

Mathematical modeling is a critical instrument that can be used in analyzing the epidemiological characteristics and dynamics. So far a wide variety of scientific models have been proposed for modeling the spread of infectious diseases. Also a good amount of research has been done for examining MERS. The transmission chains of MERS-CoV contamination in South Korea is characterized by modeling [23]. The expected measure of MERS clusters and the number of ages by employing a stochastic epidemic demonstrate is assessed [24]. A MERS-CoV transmission demonstrate with file and auxiliary cases is considered.

Mathematical Models and applications can be applied for predicting the spread of novel corona virus disease and to evaluate the possible risks as well as intervention strategies [25-27]. Based on the results of modeling and simulation studies, it is possible to recommend effective and efficient epidemiological and social techniques so as to avoid or to control the spread of the pandemic diseases.

The early models center on the MERS disease infection have considered camels as the most operator of the transmission. In those models the fundamental reproductive number $R_{0}$ is derived following the era approach. Whenever $R_{0}$ is greater than one, the disease will spread resulting in an epidemic.

Here, it is appropriate to introduce some terms and terminologies generally used in relation with corona virus COVID-19.

Community spread: A disease is said to be community spread if the disease infects people of that community without any history of contact with someone who has already infected with the disease. Containment: It refers to limiting the spread of infection by closing boundaries of some area. This procedure limits movement of the people in and out of that area. Since no antibodies exist to avoid COVID-19 and no particular treatments exist to treat it, control is done by containment. Epidemic: A disease outbreak in a community or region is called Epidemic.

Flattening the curve: It refers to the epidemic bend. Also, it is a statistical chart utilized to imagine the number of modern cases over a given period of time amid a disease outbreak. Incubation period: An exposed individual may not show up symptoms rapidly. It will take some time. The time period between exposure and appearance of symptoms is known as Incubation period.

Isolation: The infected individuals will become infectious during course of time. To avoid propagation of the disease the infectious individuals will be separated from rest of the community. This separation is termed as isolation. Mitigation: It refers to the steps initiated so as to limit spread of the disease. Since no antibodies exist to prevent COVID-19 so far, the mitigation in this case includes: Avoid visiting of places, washing hands thoroughly, not touching face, staying away from individuals who are sick, maintaining social distancing, avoiding large gatherings, and regularly cleaning frequently touched surfaces and objects.

Pandemic: It is an infection outbreak influencing huge populations or a whole locality, or a nation, or a continent or even whole world. Quarantine: It is a separation and restriction of the movements of individuals who have infectious illness. If an individual has the history of moving in the infected areas or contacting infected persons or in case become sick, then such an individual will be quarantined. Self-quarantine: It is a voluntary isolation at home by those who may have been exposed to the infection though they do not experience and exhibit any symptoms.

Symptoms: The main symptoms of COVID-19 are fever, cough, and difficulty in breathing. Self-isolation: It is a voluntary separation at home by those who are or are likely to have experiencing mild side effects of the illness. However, the extremely sick persons may be confined in a hospital. $S A R S-C o V-2$ : It is the short form for Serious Acute Respiratory Disorder - Corona Virus 2. Physical distancing: It refers to maintain enough distance between any two persons so as to reduce the risk of breathing in droplets that are produced when an infected person coughs or sneezes. In a community, it includes limiting or cancelling large gatherings of people. Virus: A virus is the smallest of infectious microbes, smaller than bacteria or fungi. A virus consists of a small piece of genetic material surrounded by a protein shell.

\section{Model Formulation}

In this study, a new mathematical model is developed for examining the transmission dynamics of COVID-19 considering human-to-human transformation of the infection. In this model, based on the status of the infection the total human population $N$ is divided into five compartments. Names and notations of these compartments are as follows: Susceptible $S$, Exposed $E$, Infected $I$, Hospitalized for laboratory tests $H_{L}$ and Hospitalized for isolation $H_{I}$.

Individuals by adding non-pharmaceutical countermeasure is called self-protection such as stay at home, keeping physical distance which is an action taken to minimize contact with other individuals, washing your hands by soap repeatedly, using sanitizer; aimed at reducing disease transmission and because of that also reducing pressure on health services on susceptible individuals and isolation (monitoring) of contacts and suspected cases on exposed and infected individuals [28].

\subsection{Model Assumptions}

The proposed model mainly based on the following assumptions: The time dependent parameter notations: (i) $\mathrm{S}(\mathrm{t})$ denotes the number of people in the susceptible compartment 
where the people are capable of infected, (ii) $E(t)$ denotes the number of people in the exposed compartment are in the incubation period; they do not show symptoms but are still capable of infecting others. (iii) $\mathrm{I}(\mathrm{t})$ denotes the number of people in the infected compartment where the people are infected with the COVID-19 (iv) $\mathrm{H}_{\mathrm{L}}(\mathrm{t})$ is the number of people in the Hospital for laboratory compartment and (v) $\mathrm{H}_{\mathrm{I}}(\mathrm{t})$ denotes the number of people in the Hospital for isolation compartment transported from the infected and exposed compartments. The transmission of COVID-19 is considered as the time independent parameter: $\mu$ represents the natural death rate or equivalently the birth rate of the population during the short span of the epidemic outbreak, $\beta$ represents the transmission rate of the disease that is the rate of transferring people from the compartment $S(t)$ to $E(t), \mathrm{f}$ represents the self- protection coefficient it reduce probability of being infected, $1 / \rho$ is the incubation period between the infection and the onset of symptoms, $1 / \delta$ is the mean time from infected of symptoms onset to Hospital for laboratory, $\mathrm{m}_{2}$ and $\mathrm{m}_{1}$ are the probabilities of individual isolated from exposed and infected compartments respectively, $\alpha$ is the rate of hospital for isolation from hospital for lab, $\gamma$ is the rate of hospital for laboratory from hospital for isolation, $\theta$ is the rate of recovery from infection mix with susceptible compartment. The above description leads to the compartmental diagram in Figure 1.

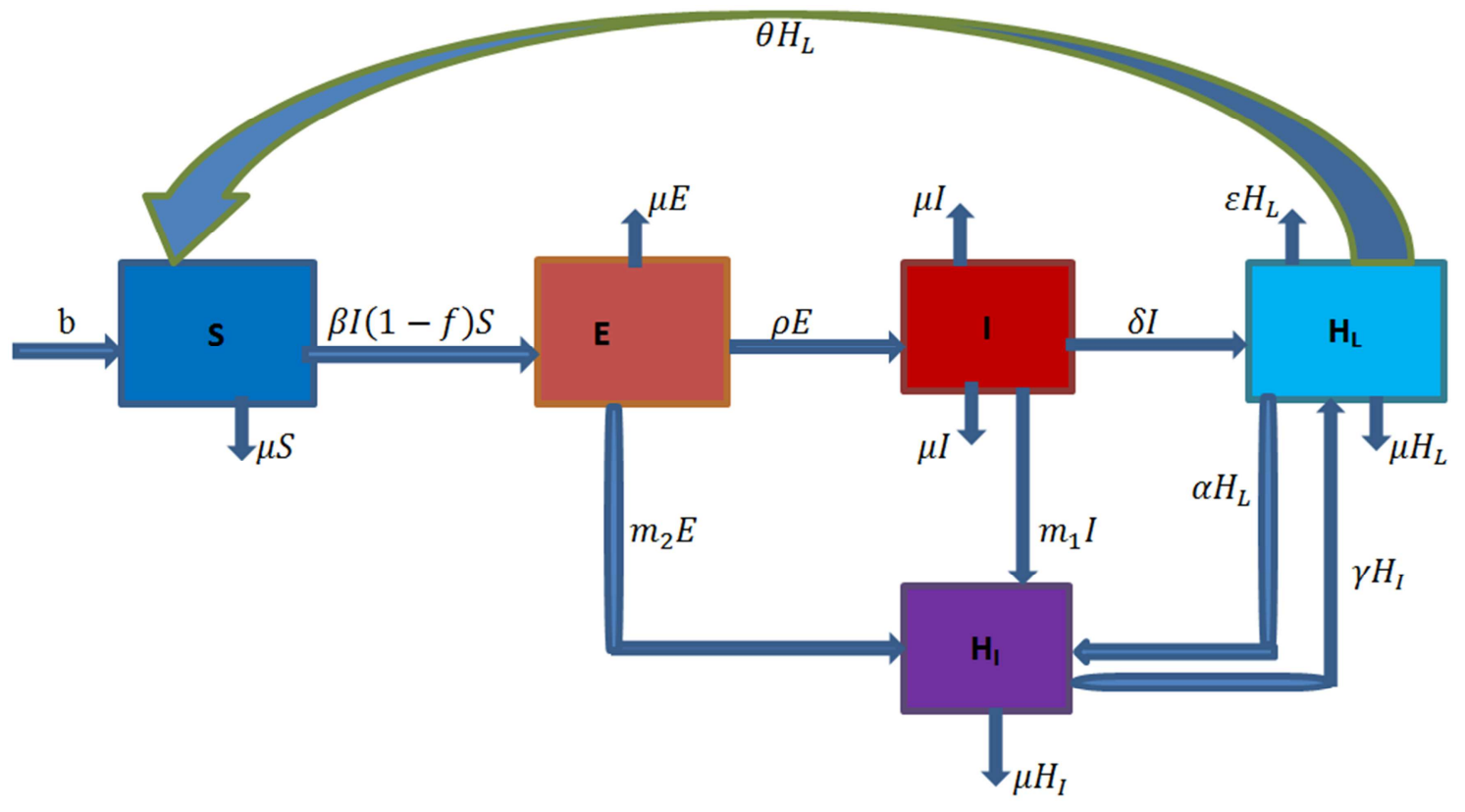

Figure 1. Compartmental diagram for COVID-19 model with control strategies.

Thus, the governing non-linear system of differential equation represent the model is given by

$$
\left.\begin{array}{c}
\frac{d S}{d t}=b+\theta H_{L}-\beta I(1-f) S-\mu S \\
\frac{d E}{d t}=\beta I(1-f) S-\left(\rho+m_{2}+\mu\right) E \\
\frac{\mathrm{dI}}{\mathrm{dt}}=\rho \mathrm{E}-\left(\delta+\mathrm{m}_{1}+\mu\right) \mathrm{I} \\
\frac{d H_{L}}{d t}=\delta I+\gamma H_{I}-(\alpha+\mu+\theta) H_{L} \\
\frac{d H_{I}}{d t}=\alpha H_{L}+m_{1} I+m_{2} E-(\gamma+\mu+\varepsilon) H_{I}
\end{array}\right\}
$$

The model variables and their description are tabulated in the table1. Model variables and parameter and their description are tabulated in the tables $1 \& 2$ respectively.

Table 1. Model variable and their description.

\begin{tabular}{ll}
\hline Variables & Description of the variables \\
\hline $\mathrm{S}(\mathrm{t})$ & Susceptible population at time $\mathrm{t}$ \\
$\mathrm{E}(\mathrm{t})$ & Exposed population at time $\mathrm{t}$ \\
$\mathrm{I}(\mathrm{t})$ & Infected population at time $\mathrm{t}$ \\
$\mathrm{H}_{\mathrm{L}}(\mathrm{t})$ & Population in hospital for laboratory test at time $\mathrm{t}$ \\
$\mathrm{H}_{\mathrm{I}}(\mathrm{t})$ & Population in hospital for isolation cases at time $\mathrm{t}$ \\
\hline
\end{tabular}

Table 2. Model parameter and their description.

\begin{tabular}{ll}
\hline Parameter & Description \\
\hline$b$ & Constant human recruitment rate \\
$\beta$ & transmission probability per contact rate \\
$f$ & Self-protection coefficient. \\
$\rho$ & Incubation period \\
$\delta$ & Infection period \\
$\Theta$ & Recovery rate of infected \\
$\mu$ & Natural human mortality rate \\
$\varepsilon$ & Disease induced death rate \\
$\mathrm{m}_{2}$ & Isolation rate from $\mathrm{I}$ to $\mathrm{H}_{\mathrm{I}}$ \\
$\mathrm{m}_{1}$ & Isolation rate from $\mathrm{E}$ to $\mathrm{H}_{\mathrm{I}}$ \\
$\alpha$ & The rate from $\mathrm{H}_{\mathrm{L}}$ to $\mathrm{H}_{\mathrm{I}}$ \\
$\gamma$ & The rate from $\mathrm{H}_{\mathrm{I}}$ to $\mathrm{H}_{\mathrm{L}}$ \\
\hline
\end{tabular}

\subsection{Positivity Solution of the Model}

Theorem 1: Let the initial conditions of system (1) $\mathrm{S}(0) \geq 0 ; \mathrm{E}(0) \geq 0 ; \mathrm{I}(0) \geq 0 ; \mathrm{H}_{\mathrm{L}}(0) \geq 0 ; \mathrm{H}_{\mathrm{I}}(0) \geq 0$, Then the solutions $\mathrm{S}(\mathrm{t}) ; \mathrm{E}(\mathrm{t}) ; \mathrm{I}(\mathrm{t}) ; \mathrm{H}_{\mathrm{L}}(\mathrm{t}), \mathrm{H}_{\mathrm{I}}(\mathrm{t})$ of the model system (1) are positively invariant for all $t \geq 0$.

Proof: Assume that all the state variables are continuous. Then, from the system (1) of the first equation one can easily 
obtained that:

$$
\frac{d S}{d t}=b+\theta H_{L}-\beta I(1-f) S-\mu S
$$

By separation of variables $\frac{1}{S} \mathrm{dS}=-(\beta \mathrm{I}(1-\mathrm{f})+\mu) \mathrm{dt}$ Integrating both sides $\int \frac{1}{S} \mathrm{~d} S=-\int(\beta \mathrm{I}(1-\mathrm{f})+\mu) \mathrm{dt}$

$$
\begin{array}{r}
\ln S=-(\beta I(1-f)+\mu) t+c \\
S(t)=S(0) e^{-(\beta I(1-f)+\mu) t}
\end{array}
$$

Since all parameters and variables are non-negative, from equation (2) we simplifying to obtain

$$
S(t) \geq S(0) e^{-(\beta I(1-f)+\mu) t} \geq 0 .
$$

Similarly;

$$
\begin{aligned}
\mathrm{E}(\mathrm{t}) & \geq \mathrm{E}(0) \mathrm{e}^{-\left(\rho+\mathrm{m}_{2}+\mu\right) \mathrm{t}} \geq 0 \\
\mathrm{I}(\mathrm{t}) & \geq \mathrm{I}(0) \mathrm{e}^{-\left(\delta+\mathrm{m}_{1}+\mu\right) \mathrm{t}} \geq 0 \\
\mathrm{H}_{\mathrm{L}}(\mathrm{t}) & \geq \mathrm{H}_{\mathrm{L}}(0) \mathrm{e}^{-(\alpha+\mu+\theta) \mathrm{t}} \geq 0 \\
\mathrm{H}_{\mathrm{I}}(\mathrm{t}) & \geq \mathrm{H}_{\mathrm{I}}(0) \mathrm{e}^{-(\gamma+\mu+\varepsilon) \mathrm{t}} \geq 0
\end{aligned}
$$

Therefore, the solutions $\mathrm{S}(\mathrm{t}) ; \mathrm{E}(\mathrm{t}) ; \mathrm{I}(\mathrm{t}) ; \mathrm{H}_{\mathrm{L}}(\mathrm{t}), \mathrm{H}_{\mathrm{I}}(\mathrm{t})$ of the model system (1) are positively invariant for all $t \geq 0$.

\subsection{Invariant Region}

The model under consideration monitors population as such; we assume that all the variables and parameters of the model are positive for all $t \geq 0$. The rate of change of the balance equations.

$$
\mathrm{N}(\mathrm{t})=\mathrm{S}(\mathrm{t})+\mathrm{E}(\mathrm{t})+\mathrm{I}(\mathrm{t})+\mathrm{H}_{\mathrm{L}}(\mathrm{t})+\mathrm{H}_{\mathrm{I}}(\mathrm{t})
$$

Differentiate both sides with respect to time t.

$$
\begin{gathered}
\frac{d N}{d t}=\frac{d S}{d t}+\frac{d E}{d t}+\frac{d I}{d t}+\frac{d H_{L}}{d t}+\frac{d H_{I}}{d t} \\
=b-\mu\left(S+E+I+H_{L}+H_{I}\right)-\varepsilon H_{I} \\
=b-\mu N-\varepsilon H_{I} \\
\frac{d N}{d t} \leq b-\mu N
\end{gathered}
$$

Implying $\frac{d N(t)}{d t}$ is bounded above by $b-\mu N(t)$.

By separation of variables of differential inequality method (9) becomes:

$$
\mathrm{b}-\mu \mathrm{N} \geq \mathrm{Ce}^{-\mu \mathrm{t}}
$$

Where $\mathrm{C}$ is constant, hence at $\mathrm{t}=0, \mathrm{~N}(0)=\mathrm{N}_{0}$, implies $C=\mathrm{b}-\mu \mathrm{N}_{0}$. Then substitute the value of $\mathrm{C}$.

$$
\begin{aligned}
\mathrm{b}-\mu \mathrm{N} & \geq\left(\mathrm{b}-\mu \mathrm{N}_{0}\right) \mathrm{e}^{-\mu \mathrm{t}}, \\
\mathrm{N} & \leq \mathrm{b}-\left(\frac{\mathrm{b}-\mu \mathrm{N}_{0}}{\mu}\right) \mathrm{e}^{-\mu \mathrm{t}},
\end{aligned}
$$

$$
0 \leq \mathrm{N} \leq \mathrm{b} \text {, then } \lim _{\mathrm{t} \rightarrow \infty} \sup \mathrm{N}(\mathrm{t}) \leq \frac{\mathrm{b}}{\mu}
$$

Therefore, the feasible solutions set of system (1) enters and remain in region $\Omega$ for all time $t$,

$$
\Omega=\left\{\left(\mathrm{S}, \mathrm{E}, \mathrm{I}, \mathrm{H}_{\mathrm{L}}, \mathrm{H}_{\mathrm{I}}\right) \in \mathbb{R}_{+}^{5} ; \mathrm{S}, \mathrm{E}, \mathrm{I}, \mathrm{H}_{\mathrm{L}}, \mathrm{H}_{\mathrm{I}} \geq 0 ; \mathrm{N}(\mathrm{t}) \leq \frac{\mathrm{b}}{\mu}\right\}(
$$

This is a positively invariant set under the flow induced by the model system (1). Hence the system is biologically meaningful and mathematically well-posed in the domain $\Omega$.

\subsection{Determination of basic Reproduction Number}

The basic reproductive number $R_{0}$ is defined as the expected number of new infected from one infected individual in a fully susceptible population through the entire duration of infection prone period. We use the next generation operator approach as described by [29] to define the basic reproduction number $\mathrm{R}_{0}$, as the number of secondary infections that one infections individual would create over the duration of the infectious period, provided that everyone else is susceptible. Basic reproduction number $\mathrm{R}_{0}$ is the threshold for many epidemiology models, it determines whether a disease can invade in the population or not. When $\mathrm{R}_{0}<1$ each infected individual produces on average less than one new infect individual. So we would expect that the disease to minimize. On the other hand, if $\mathrm{R}_{0}>1$, each infected individual produces new infect individual, so we would expect that the disease to spread in the population. This means that the threshold quantity for eradicating the disease is to reduce the value of $R_{0}$ to value less than one.

Let

1) $\mathcal{F}_{\mathrm{i}}(\mathrm{x})$ be the rate of appearance of new infect in compartment i,

2) $\mathcal{V}_{\mathrm{i}}^{+}(\mathrm{x})$ is the rate of individuals transfer into compartment $i$,

3) $\mathcal{V}_{\mathrm{i}}^{-}(\mathrm{x})$ is the rate of individual transfer out of the compartment $i$.

From system (1) the infected compartments to be

$$
\left.\begin{array}{c}
\frac{\mathrm{dE}}{\mathrm{dt}}=\beta \mathrm{I}(1-\mathrm{f}) \mathrm{S}-\left(\rho+\mathrm{m}_{2}+\mu\right) \mathrm{E} \\
\frac{\mathrm{dI}}{\mathrm{dt}}=\rho \mathrm{E}-\left(\delta+\mathrm{m}_{1}+\mu\right) \mathrm{I} \\
\frac{\mathrm{dH}_{\mathrm{L}}}{\mathrm{dt}}=\delta \mathrm{I}+\gamma \mathrm{H}_{\mathrm{I}}-(\alpha+\mu+\theta) \mathrm{H}_{\mathrm{L}} \\
\frac{\mathrm{dH}_{\mathrm{I}}}{\mathrm{dt}}=\alpha \mathrm{H}_{\mathrm{L}}+\mathrm{m}_{1} \mathrm{I}+\mathrm{m}_{2} \mathrm{E}-(\gamma+\mu+\varepsilon) H_{I}
\end{array}\right\}
$$

From system (14), we define $\mathcal{F}_{\mathrm{i}}$ and $\mathcal{V}_{\mathrm{i}}$ as

$$
\begin{aligned}
\mathcal{F}_{\mathrm{i}}=\left(\begin{array}{c}
\beta \mathrm{I}(1-\mathrm{f}) \mathrm{S} \\
0 \\
0 \\
0
\end{array}\right), \mathrm{F}=\frac{\partial \mathcal{F}_{\mathrm{i}}}{\partial \mathrm{x}_{\mathrm{i}}} & \left(\begin{array}{rrrr}
0 & \beta(1-\mathrm{f}) \mathrm{S} & 0 & 0 \\
0 & 0 & 0 & 0 \\
0 & 0 & 0 & 0 \\
0 & 0 & 0 & 0
\end{array}\right), \quad \mathrm{F}_{\epsilon_{0}}= \\
& \left(\begin{array}{cccc}
0 & \frac{\beta(1-f) b}{\mu} & 0 & 0 \\
0 & 0 & 0 & 0 \\
0 & 0 & 0 & 0 \\
0 & 0 & 0 & 0
\end{array}\right)
\end{aligned}
$$




$$
\mathcal{V}_{\mathrm{i}}=\mathcal{V}_{\mathrm{i}}^{-}-\mathcal{V}_{\mathrm{i}}^{+}=\left(\begin{array}{c}
\left(\rho+\mathrm{m}_{2}+\mu\right) \mathrm{E} \\
\left(\delta+\mathrm{m}_{1}+\mu\right) \mathrm{I}-\rho \mathrm{E} \\
(\alpha+\mu+\theta) \mathrm{H}_{\mathrm{L}}-\delta \mathrm{I}-\gamma \mathrm{H}_{\mathrm{I}} \\
(\gamma+\mu+\varepsilon) \mathrm{H}_{\mathrm{I}}-\alpha \mathrm{H}_{\mathrm{L}}-\mathrm{m}_{1} \mathrm{I}-\mathrm{m}_{2} \mathrm{E}
\end{array}\right)
$$

Where

$$
\begin{aligned}
& v_{\mathrm{i}}^{-}=\left(\begin{array}{c}
\left(\rho+\mathrm{m}_{2}+\mu\right) \mathrm{E} \\
\left(\delta+\mathrm{m}_{1}+\mu\right) \mathrm{I} \\
(\alpha+\mu+\theta) \mathrm{H}_{\mathrm{L}} \\
(\gamma+\mu+\varepsilon) \mathrm{H}_{\mathrm{I}}
\end{array}\right) \text {, and } \mathcal{V}_{\mathrm{i}}^{+}=\left(\begin{array}{c}
0 \\
\rho \mathrm{E} \\
\delta \mathrm{I}+\gamma \mathrm{H}_{\mathrm{I}} \\
\alpha \mathrm{H}_{\mathrm{L}}+\mathrm{m}_{1} \mathrm{I}+\mathrm{m}_{2} \mathrm{E}
\end{array}\right) \\
& \mathrm{V}_{\epsilon_{0}}=\frac{\partial v_{\mathrm{i}}}{\partial \mathrm{x}_{\mathrm{i}}}=\left(\begin{array}{cccc}
\left(\rho+\mathrm{m}_{2}+\mu\right) & 0 & 0 & 0 \\
-\rho & \left(\delta+\mathrm{m}_{1}+\mu\right) & 0 & 0 \\
0 & -\delta & (\alpha+\mu+\theta) & -\gamma \\
-\mathrm{m}_{2} & -\mathrm{m}_{1} & -\alpha & (\gamma+\mu+\varepsilon)
\end{array}\right)
\end{aligned}
$$

Therefore, the next generation matrix is given by:

$$
\mathrm{FV}^{-1}=\left(\begin{array}{c}
0 \\
0 \\
0 \\
\beta(1-\mathrm{f}) \rho \mathrm{b} \\
\frac{\mu\left(\delta \rho+\delta \mathrm{m}_{2}+\delta \mu+\mathrm{m}_{1} \rho+\mathrm{m}_{1} \mathrm{~m}_{2}+\mathrm{m}_{1} \mu+\mu \rho+\mu \mathrm{m}_{2}+\mu^{2}\right)}{{ }^{2}}
\end{array}\right)
$$

Basic reproduction number, $\mathrm{R}_{0}=\rho\left(\mathrm{FV}^{-1}\right)$, where is the spectral radius (maximum eigenvalue) of the matrix of the model.

$$
\mathrm{R}_{0}=\frac{\beta(1-\mathrm{d}) \rho \mathrm{b}}{\mu\left(\rho+\mathrm{m}_{2}+\mu\right)\left(\mathrm{m}_{1}+\mu+\delta\right)}
$$

\section{Stability Analysis}

\subsection{Existence of the Disease Free Equilibrium Point}

The disease free equilibrium point $\left(\epsilon_{0}\right)$ is the state in which the population is frees of disease that $\mathrm{E}=0, \mathrm{I}=$ $0, \mathrm{H}_{\mathrm{L}}=0$ and $\mathrm{H}_{\mathrm{I}}=0$. thus, the model has a disease free

$$
\begin{aligned}
& \mathrm{J}_{\epsilon_{0}}=\left(\begin{array}{ccccc}
-\mu & 0 & -\beta(1-f) S^{*} & \theta & 0 \\
0 & -\left(\rho+\mathrm{m}_{2}+\mu\right) & \beta(1-f) S^{*} & 0 & 0 \\
0 & \rho & -\left(\mathrm{m}_{1}+\mu+\delta\right) & 0 & 0 \\
0 & 0 & \delta & -(\alpha+\mu+\theta) & \gamma \\
0 & \mathrm{~m}_{2} & \mathrm{~m}_{1} & \alpha & -(\gamma+\mu+\varepsilon)
\end{array}\right) \\
& \left|\mathrm{J}_{\epsilon_{0}}\right|=\left|\begin{array}{ccccc}
-(\mu) & 0 & -\frac{\beta(1-\mathrm{f}) \mathrm{b}}{\mu} & \theta & 0 \\
0 & -\left(\rho+\mathrm{m}_{2}+\mu\right) & \frac{\beta(1-\mathrm{f}) \mathrm{b}}{\mu} & 0 & 0 \\
0 & \rho & -\left(\mathrm{m}_{1}+\mu+\delta\right) & 0 & 0 \\
0 & 0 & \delta & \alpha & -(\alpha+\mu+\theta) \\
0 & \mathrm{~m}_{2} & \mathrm{~m}_{1} & & \gamma
\end{array}\right|
\end{aligned}
$$

Let, $\mathrm{a}_{1}=\left(\rho+\mathrm{m}_{2}+\mu\right), \mathrm{a}_{2}=\frac{\beta(1-\mathrm{f}) \mathrm{b}}{\mu}, \mathrm{a}_{3}=\left(\mathrm{m}_{1}+\mu+\delta\right), \mathrm{a}_{4}=(\alpha+\mu+\theta), \mathrm{a}_{5}=(\gamma+\mu+\varepsilon)$

Hence characteristic equation of $\left|J_{\epsilon_{0}}-\lambda I_{5}\right|=0$ is;

$$
\begin{gathered}
\left|\mathrm{J}_{\epsilon_{0}}-\lambda \mathrm{I}_{5}\right|=\left|\begin{array}{ccccc}
-(\mu+\lambda) & 0 & -\mathrm{a}_{2} & \theta & 0 \\
0 & -\left(\mathrm{a}_{1}+\lambda\right) & \mathrm{a}_{2} & 0 & 0 \\
0 & \rho & -\left(\mathrm{a}_{3}+\lambda\right) & 0 & 0 \\
0 & 0 & \delta & -\left(\mathrm{m}_{4}+\lambda\right) & \gamma \\
0 & \mathrm{~m}_{2} & \mathrm{~m}_{1} & \alpha & -\left(\mathrm{m}_{5}+\lambda\right)
\end{array}\right|=0 \\
=-(\mu+\lambda)\left[-\gamma \alpha\left(\left(\mathrm{a}_{1}+\lambda\right)\left(\mathrm{a}_{3}+\lambda\right)-\mathrm{a}_{2} \rho\right)+\left(\mathrm{m}_{5}+\lambda\right)\left(\mathrm{m}_{4}+\lambda\right)\left(\left(\mathrm{a}_{1}+\lambda\right)\left(\mathrm{a}_{3}+\lambda\right)-\mathrm{a}_{2} \rho\right)\right]
\end{gathered}
$$




$$
=-(\mu+\lambda)\left[\left(\left(a_{1}+\lambda\right)\left(a_{3}+\lambda\right)-a_{2} \rho\right)\right]\left[\left(m_{5}+\lambda\right)\left(m_{4}+\lambda\right)-\gamma \alpha\right]=0
$$

We have eigenvalues

$$
-(\mu+\lambda)=0 \Rightarrow \lambda_{1}=-\mu<0,
$$

$\left(\left(a_{1}+\lambda\right)\left(a_{3}+\lambda\right)-a_{2} \rho\right)=0$, From this quadratic equation we have eigenvalues

$$
\lambda_{2,3}=-\frac{\left(\rho+\mathrm{m}_{2}+2 \mu+\mathrm{m}_{1}+\delta\right)}{2} \pm \frac{1}{2} \sqrt{\begin{array}{c}
\delta^{2}+\left(2 \mathrm{~m}_{1}-2 \mathrm{~m}_{2}-2 \rho\right) \delta+\mathrm{m}_{1}{ }^{2}+\left(-2 \rho-2 \mathrm{~m}_{2}\right) \mathrm{m}_{1} \\
+\mathrm{m}_{2}{ }^{2}+2 \mathrm{~m}_{2} \rho+\rho^{2}+4 \beta(1-\mathrm{f}) \mathrm{b}
\end{array}}
$$

$\left(m_{5}+\lambda\right)\left(m_{4}+\lambda\right)-\gamma \alpha=0$, Again from this quadratic equation we have eigenvalues

$$
\lambda_{4,5}=-\frac{\left(\rho+\mathrm{m}_{2}+\mu+\mathrm{m}_{1}+\delta\right)}{2} \pm \frac{1}{2} \sqrt{\begin{array}{c}
\left(\mathrm{m}_{1}+\delta\right)\left(\mathrm{m}_{1}+\delta\right)-2 \delta\left(\rho+\mathrm{m}_{2}\right)+\mathrm{m}_{1}^{2}-2\left(\rho+\mathrm{m}_{2}\right) \\
\mathrm{m}_{1}+\left(\rho+\mathrm{m}_{2}\right)\left(\rho+\mathrm{m}_{2}\right)+4 \beta(1-\mathrm{f}) \mathrm{b}
\end{array}}
$$

Routh-Hurwitz criteria give necessary and sufficient condition for the negativity of the roots (eigenvalues) of quadratic equation and since all parameter are positive the disease free equilibrium point is locally asymptotically stable if $\mathrm{R}_{0}<1$.

\subsection{Global Stability of the Disease Free Equilibrium Point}

In this section, we study the global properties of the disease-free equilibrium. The following theorem provides the global property of the disease free equilibrium:

Theorem 3: The disease free equilibrium, $\left(\epsilon_{0}\right)$ of the

$$
\begin{aligned}
& \mathbb{V}^{\prime} \leq \beta \mathrm{I}(1-\mathrm{f}) \mathrm{S}^{*}-\left(\rho+\mathrm{m}_{2}+\mu\right)\left(\frac{\left(\delta+\mathrm{m}_{1}+\mu\right) \mathrm{I}}{\rho}\right)+\rho\left(\frac{\left(\delta+\mathrm{m}_{1}+\mu\right) \mathrm{I}}{\rho}\right)-\left(\delta+\mathrm{m}_{1}+\mu\right) \mathrm{I} \\
& \text { Since } \mathrm{S} \leq \mathrm{S}^{*}, \text { and } \mathrm{E}=\frac{\left(\delta+\mathrm{m}_{1}+\mu\right)}{\rho} \mathrm{I} \\
& \mathbb{V}^{\prime}=\mathrm{I}\left(\frac{\left(\rho+\mathrm{m}_{2}+\mu\right)\left(\delta+\mathrm{m}_{1}+\mu\right)}{\rho}\right)\left(\frac{\beta(1-\mathrm{f}) \rho \mathrm{b}}{\mu\left(\rho+\mathrm{m}_{2}+\mu\right)\left(\delta+\mathrm{m}_{1}+\mu\right)}-1\right) \\
& \mathbb{V}^{\prime}=\mathrm{I}\left(\frac{\left(\rho+\mathrm{m}_{2}+\mu\right)\left(\delta+\mathrm{m}_{1}+\mu\right)}{\rho}\right)\left(\mathrm{R}_{0}-1\right)
\end{aligned}
$$

Since all parameter and variables are non-negative we obtain $\mathbb{V}^{\prime} \leq 0$ for $\mathrm{R}_{0} \leq 1$. Hence, by [30] every solution that starts in the region $\Omega$ approaches $\left(\epsilon_{0}\right)$ as $t \rightarrow \infty$ is over all globally asymptotically stable for $R_{0} \leq 1$ in region $\mathbb{R}_{+}^{5}$.

\subsection{Existence of the Disease Endemic Equilibrium Point}

Endemic equilibrium point $\left(\epsilon_{1}\right)$ is a steady state solution where the disease persists in the population. Hence we solve the system (1) of equations at steady-state which gives;

$$
\begin{gathered}
S^{1}=\frac{b}{\mathrm{R}_{0} \gamma} \\
\mathrm{E}^{1}=\frac{\mathrm{b} \theta}{\mathrm{R}_{0} \gamma \rho^{3}}\left[\delta+\mathrm{m}_{1}+\mu\right]^{2}\left[\mathrm{R}_{0}-1\right][(\gamma+\mu+\varepsilon)(\alpha+\mu+\theta)-\alpha]^{2} \\
{\left[\rho\left(\mathrm{m}_{1} \gamma+\delta(\gamma+\mu+\varepsilon)\right) \mathrm{m}_{2} \gamma\left(\delta+\mathrm{m}_{1}+\mu\right)\right]\left[\rho+\mathrm{m}_{2}+\mu\right]} \\
\mathrm{I}^{1}=\frac{\mathrm{b} \theta}{\mathrm{R}_{0} \gamma \rho^{2}}[(\gamma+\mu+\varepsilon)(\alpha+\mu+\theta)-\alpha]^{2}\left[\mathrm{R}_{0}-1\right]\left[\rho\left(\mathrm{m}_{1} \gamma+\delta(\gamma+\mu+\varepsilon)\right) \mathrm{m}_{2} \gamma\left(\delta+\mathrm{m}_{1}+\mu\right)\right] \\
{\left[\rho+\mathrm{m}_{2}+\mu\right]\left[\delta+\mathrm{m}_{1}+\mu\right]}
\end{gathered}
$$




$$
\begin{aligned}
& \mathrm{H}_{\mathrm{L}}{ }^{1}=\frac{\frac{\mathrm{b}}{\mathrm{R}_{0}}\left[\mathrm{R}_{0}-1\right]\left[(\delta \mu \rho)+\gamma \mathrm{m}_{2}{ }^{2}+\gamma(\delta+\mu) \mathrm{m}_{2}+\rho\left((\mu+\varepsilon) \delta+\gamma \mathrm{m}_{1}\right)\right]}{\left[\begin{array}{c}
\left(\delta+\mathrm{m}_{1}+\mu\right)\left(\rho+\mathrm{m}_{2}+\mu\right) \mu^{2}+\left((\gamma+\theta+\varepsilon+\alpha) \mathrm{m}_{2}{ }^{2}+2\left(\mu+\frac{1}{2} \rho+\frac{1}{2} \delta\right)(\gamma+\theta+\varepsilon+\alpha) \mathrm{m}_{2}+(\gamma+\theta+\varepsilon+\alpha) \mu^{2}\right. \\
+(\delta+\rho)(\gamma+\theta+\varepsilon+\alpha) \mu+\delta \rho(\gamma+\varepsilon+\alpha)) \mu+\varepsilon(\theta+\alpha) \mathrm{m}_{2}{ }^{2}+(((2 \theta+2 \alpha) \varepsilon+\gamma \theta) \mu+(\varepsilon(\theta+\alpha)+\gamma \theta) \rho \\
\left.+\varepsilon \delta(\theta+\alpha) \mathrm{m}_{2}+(\varepsilon(\theta+\alpha)+\gamma \theta) \mu^{2}+(\delta+\rho)(\varepsilon(\theta+\alpha)+\gamma \theta)\right)+\rho\left(\alpha \delta \varepsilon-\gamma \mathrm{m}_{1} \theta\right)
\end{array}\right]} \\
& \mathrm{H}_{\mathrm{I}}{ }^{1}=\frac{\frac{\mathrm{b}}{\mathrm{R}_{0}}\left[\mathrm{R}_{0}-1\right]\left[(\mu+\alpha+\theta) \mathrm{m}_{2}{ }^{2}+(\delta+\mu)(\mu+\alpha+\theta) \mathrm{m}_{2}+\left(\mathrm{m}_{1} \mu+\alpha \delta+\mathrm{m}_{1}(\theta+\alpha) \rho\right)\right]}{\left[\begin{array}{c}
\left(\mu^{2}+(\gamma+\theta+\varepsilon+\alpha) \mu+\varepsilon(\theta+\alpha)\right) \mathrm{m}_{2}{ }^{2}+\left((2 \mu+\rho+\delta) \mu^{2}+2\left(\mu+\frac{1}{2} \rho+\frac{1}{2} \delta\right)(\gamma+\theta+\varepsilon+\alpha) \mu\right. \\
+((\gamma+2 \varepsilon) \theta+2 \varepsilon \alpha) \mu+((\gamma+\varepsilon) \theta+\varepsilon \alpha) \rho+\delta \varepsilon(\theta+\alpha)) \mathrm{m}_{2}+(\delta+\mu)(\delta+\mu) \mu^{2}+(\gamma+\theta+\varepsilon+\alpha) \mu^{2} \\
+(\rho+\delta)(\gamma+\theta+\varepsilon+\alpha) \mu+\delta \rho(\gamma+\varepsilon+\alpha) \mu+((\gamma+\varepsilon) \theta+\varepsilon \alpha) \mu^{2}+(\delta+\rho)((\gamma+\varepsilon) \theta+\varepsilon \alpha) \mu+\rho\left(\alpha \delta \varepsilon-\gamma \mathrm{m}_{1} \theta\right)
\end{array}\right]}
\end{aligned}
$$

\subsection{Global Stability of the Endemic Equilibrium Point}

Theorem 4: The disease endemic equilibrium, $\left(\epsilon_{1}\right)$ of the system (1) globally asymptotically stable (GAS) in region $\Omega$ if $R_{0}>1$.
Proof: suppose $R_{0}>1$; then the existence of the endemic equilibrium point is assumed using the common quadratic Lyapunov function

$$
\mathbb{L}\left(\mathrm{x}_{1}, \mathrm{x}_{2}, \mathrm{x}_{3} \ldots \mathrm{x}_{\mathrm{n}}\right)=\sum_{1}^{\mathrm{n}} \frac{\mathrm{C}_{\mathrm{i}}}{2}\left(\mathrm{x}_{\mathrm{i}}-\mathrm{x}_{\mathrm{i}}{ }^{1}\right)^{2} .
$$

Hence we consider the function

$$
\mathbb{L}\left(\mathrm{S}, \mathrm{E}, \mathrm{I}, \mathrm{H}_{\mathrm{L}}, \mathrm{H}_{\mathrm{I}}\right)=\frac{1}{2}\left(\mathrm{~S}-\mathrm{S}^{1}\right)^{2}+\frac{1}{2}\left(\mathrm{E}-\mathrm{E}^{1}\right)^{2}+\frac{1}{2}\left(\mathrm{I}-\mathrm{I}^{1}\right)^{2}+\frac{1}{2}\left(\mathrm{H}_{\mathrm{L}}-\mathrm{H}_{\mathrm{L}}{ }^{1}\right)^{2}+\frac{1}{2}\left(\mathrm{H}_{\mathrm{I}}-\mathrm{H}_{\mathrm{I}}{ }^{1}\right)^{2}
$$

Its derivatives along the solutions of the model equations is:

$$
\begin{gathered}
\mathbb{L}^{\prime}=\left[\left(S-\mathrm{S}^{1}\right)+\left(\mathrm{E}-\mathrm{E}^{1}\right)+\left(\mathrm{I}-\mathrm{I}^{1}\right)+\left(\mathrm{H}_{\mathrm{L}}-\mathrm{H}_{\mathrm{L}}{ }^{1}\right)+\left(\mathrm{H}_{\mathrm{I}}-\mathrm{H}_{\mathrm{I}}{ }^{1}\right)\right]\left[\frac{\mathrm{dS}}{\mathrm{dt}}+\frac{\mathrm{dE}}{\mathrm{dt}}+\frac{\mathrm{dI}}{\mathrm{dt}}+\frac{\mathrm{dH}_{\mathrm{L}}}{\mathrm{dt}}+\frac{\mathrm{dH}_{\mathrm{I}}}{\mathrm{dt}}\right] \\
=\left[\left(\mathrm{S}-\mathrm{S}^{1}\right)+\left(\mathrm{E}-\mathrm{E}^{1}\right)+\left(\mathrm{I}-\mathrm{I}^{1}\right)+\left(\mathrm{H}_{\mathrm{L}}-\mathrm{H}_{\mathrm{L}}{ }^{1}\right)+\left(\mathrm{H}_{\mathrm{I}}-\mathrm{H}_{\mathrm{I}}{ }^{1}\right)\right]\left[\mathrm{b}-\mu\left(\mathrm{S}+\mathrm{E}+\mathrm{I}+\mathrm{H}_{\mathrm{L}}+\mathrm{H}_{\mathrm{I}}\right)-\varepsilon \mathrm{H}_{\mathrm{I}}\right] \\
=\left[\left(\mathrm{S}-\mathrm{S}^{1}\right)+\left(\mathrm{E}-\mathrm{E}^{1}\right)+\left(\mathrm{I}-\mathrm{I}^{1}\right)+\left(\mathrm{H}_{\mathrm{L}}-\mathrm{H}_{\mathrm{L}}{ }^{1}\right)+\left(\mathrm{H}_{\mathrm{I}}-\mathrm{H}_{\mathrm{I}}{ }^{1}\right)\right] \\
{\left[\mu\left(\mathrm{S}^{1}+\mathrm{E}^{1}+\mathrm{I}^{1}+\mathrm{H}_{\mathrm{L}}{ }^{1}+\mathrm{H}_{\mathrm{I}}{ }^{1}\right)-\mu\left(\mathrm{S}+\mathrm{E}+\mathrm{I}+\mathrm{H}_{\mathrm{L}}+\mathrm{H}_{\mathrm{I}}\right)-\varepsilon \mathrm{H}_{\mathrm{I}}\right]}
\end{gathered}
$$

Where $\mathrm{b}$ is solved from total population at steady state and at equilibrium point.

$$
\begin{gathered}
\mathbb{L}^{\prime}=-\mu\left[\left(S-S^{1}\right)^{2}+\left(E-E^{1}\right)^{2}+\left(I-I^{1}\right)^{2}+\left(H_{L}-H_{L}{ }^{1}\right)^{2}+\left(H_{I}-H_{I}{ }^{1}\right)^{2}\right]-\varepsilon\left[H_{I}-H_{I}{ }^{1}\right]^{2} \\
-\mu\left[2\left(S-S^{1}\right)\left(E-E^{1}\right)+2\left(S-S^{1}\right)\left(I-I^{1}\right)+2\left(S-S^{1}\right)\left(H_{L}-H_{L}{ }^{1}\right)+2\left(S-S^{1}\right)\left(H_{I}-H_{I}{ }^{1}\right)\right] \\
-\mu\left[2\left(E-E^{1}\right)\left(I-I^{1}\right)+2\left(E-E^{1}\right)\left(H_{L}-H_{L}{ }^{1}\right)+2\left(E-E^{1}\right)\left(H_{I}-H_{I}{ }^{1}\right)\right] \\
-\mu\left[2\left(I-I^{1}\right)\left(H_{L}-H_{L}{ }^{1}\right)+2\left(I-I^{1}\right)\left(H_{I}-H_{I}{ }^{1}\right)\right]-\mu\left[2\left(H_{L}-H_{L}{ }^{1}\right)\left(H_{I}-H_{I}{ }^{1}\right)\right]
\end{gathered}
$$

Thus, shows that $\frac{\mathrm{d} \mathbb{L}}{\mathrm{dt}}$ is negative and $\frac{\mathrm{d} \mathbb{L}}{\mathrm{dt}}=0$, if and only if $\mathrm{S}=\mathrm{S}^{1}, \mathrm{E}=\mathrm{E}^{1}, \mathrm{I}=\mathrm{I}^{1}, \mathrm{H}_{\mathrm{L}}=\mathrm{H}_{\mathrm{L}}{ }^{1}$ and

$\mathrm{H}_{\mathrm{I}}=\mathrm{H}_{\mathrm{I}}{ }^{1}$ Then, the largest invariant set of system (1) on the set $\left(\mathrm{S}, \mathrm{E}, \mathrm{I}, \mathrm{H}_{\mathrm{L}}, \mathrm{H}_{\mathrm{I}}\right) \in \Omega \subset \mathbb{R}_{+}^{5}: \mathbb{L}^{\prime} \leq 0$

Is the endemic equilibrium point $\left(\epsilon_{1}\right)$. every solution to the system equation with the initial conditions in, approaches $\left(\epsilon_{1}\right)$ as $\mathrm{t} \rightarrow \infty$ if $\mathrm{R}_{0}>1$. hence, the endemic equilibrium $\left(\epsilon_{1}\right)$ is globally asymptotically stable if $\mathrm{R}_{0}>1$.

\begin{tabular}{|c|c|c|c|}
\hline Parameter & Description & Estimated mean value & Source \\
\hline$\beta$ & transmission probability per contact rate & 0.5925 & {$[16]$} \\
\hline b & Birth rate & 0.0000301 & {$[23]$} \\
\hline $\mathrm{f}$ & Self-protection rate. & $(0,1)$ & Verified \\
\hline$\mu$ & Natural mortality rate & 0.0000301 & {$[23]$} \\
\hline
\end{tabular}

\section{Simulation Study of the Model}

Here we consider simulation study of COVID-19 model with varying values of reproductive number $R_{0}$. The main focus of the simulation study is to investigate the response of model parameters up on the COVID-19 pandemic disease. We have considered $R_{0}$ assigning different values few of which are less than and the other are greater than one unit and conduct simulation study.

The simulation studies and the analysis made are based on these standard values which are displayed below in Table 3.

Table 3. Definitions and values of model parameters. 


\begin{tabular}{llll}
\hline Parameter & Description & Estimated mean value & Source \\
\hline$\delta$ & Infection rate & 0.1887 & {$[10]$} \\
$\theta$ & Recovery rate of infected & 0.0590 & {$[31]$} \\
$\rho$ & Inverse of Incubation period & 0.1783 & {$[32]$} \\
$\varepsilon$ & Disease induced death rate & 0.0300 & Calculated \\
$\mathrm{m}_{2}$ & Isolation rate from I to $\mathrm{H}_{\mathrm{I}}$ & $(0,1)$ & Verified \\
$\mathrm{m}_{1}$ & Isolation rate from $\mathrm{E}$ to $\mathrm{H}_{\mathrm{I}}$ & $(0,1)$ & Verified \\
$\alpha$ & The rate from $\mathrm{H}_{\mathrm{L}}$ to $\mathrm{H}_{\mathrm{I}}$ & 0.1971 & Assumption \\
$\gamma$ & The rate from $\mathrm{H}_{\mathrm{I}}$ to $\mathrm{H}_{\mathrm{L}}$ & 0.1200 & Assumption \\
\hline
\end{tabular}

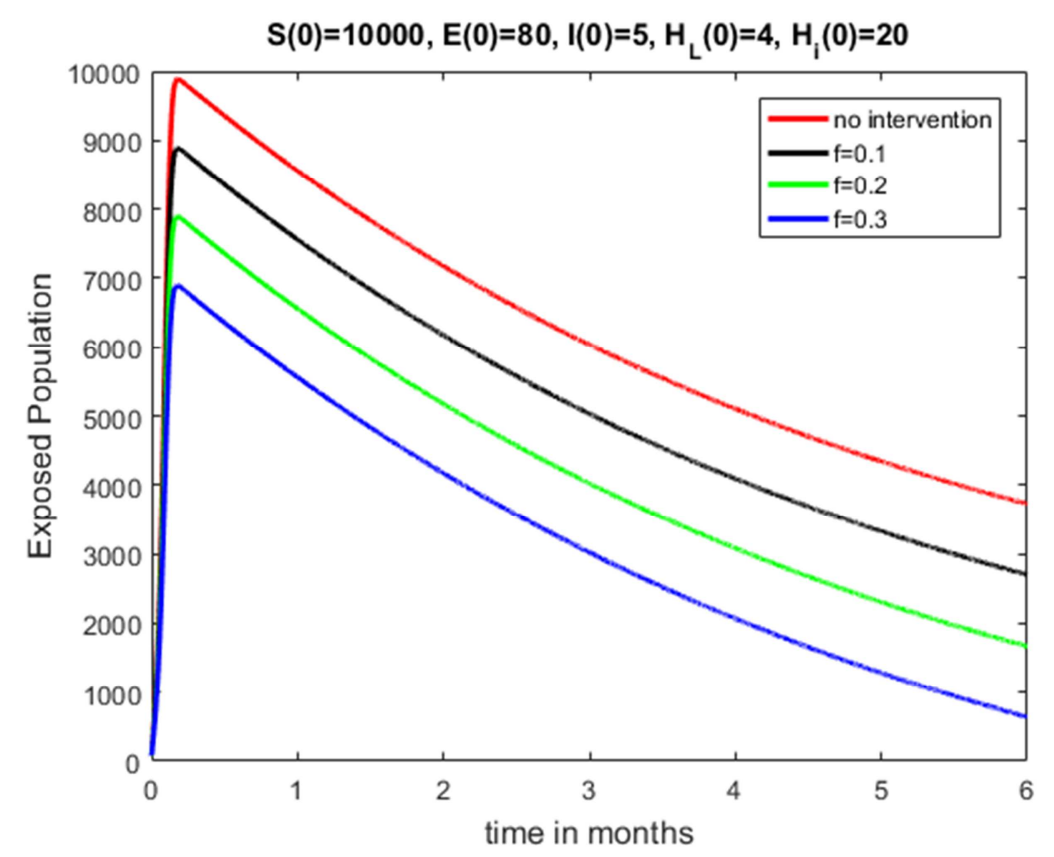

(a). Effect of self-protective on Exposed population

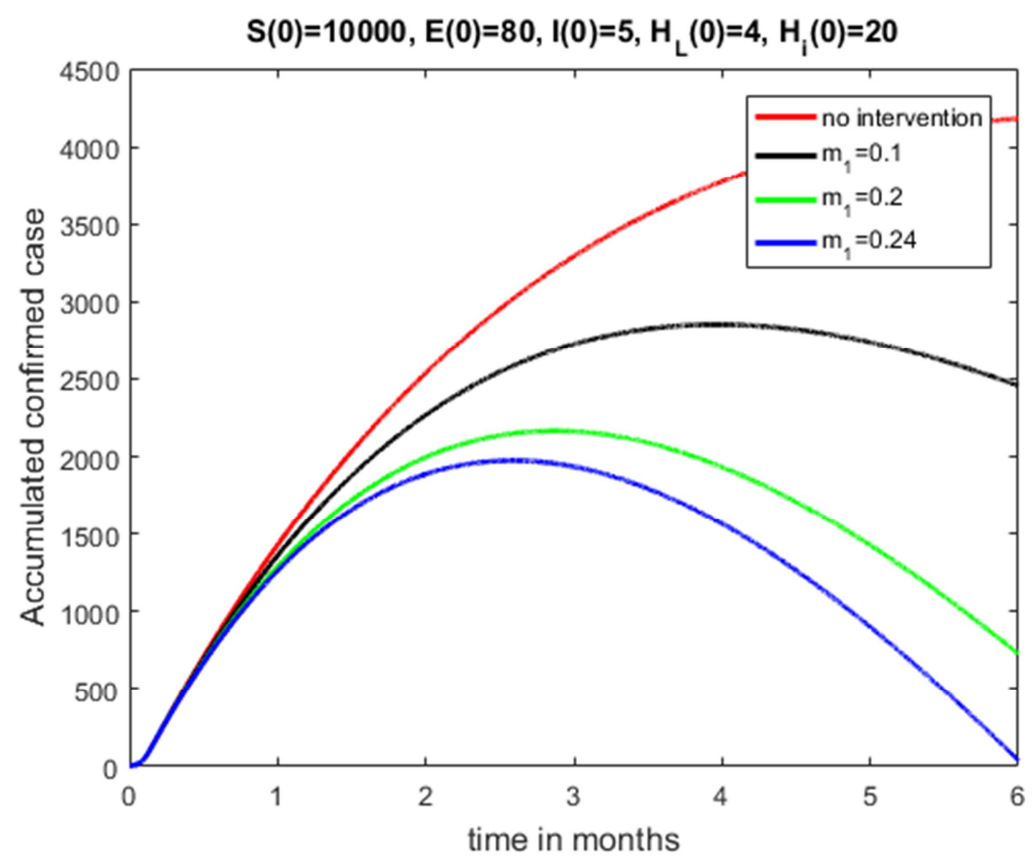

(b). Effect of isolation on Infected Population

Figure 2. Relation between intervention and Exposed and Infected compartment.

Looking at the simulated curves in Figure 2 (a), it is seen that initially when no intervention is applied the number of exposed humans is increasing and then, start decreasing. This is caused by the movement of the susceptible humans to the 
exposed compartment after being the contacts between infected and susceptible individuals. While in the exposed compartment, when the COVID-19 symptoms start to show up, the exposed humans move to the infected compartment, decreasing the exposed humans. Comparing each curves of (a) it is obvious that the bigger value the self-protective coefficient (f), the smaller the final size of exposed humans. Observing Figure 2 (b), when no intervention is applied the number of infected humans is increasing before it starts to decrease. This is due to the number of exposed humans who started showing COVID-19 symptoms moving to the infected compartment. The number of infected humans is thereafter decreasing because infected compartment moves to the hospital for laboratory and isolation compartment. Comparing each curves of (b) it is obvious that the bigger value the isolation parameter $\mathrm{m}_{1}$, the smaller the final size of accumulated confirmed cases.

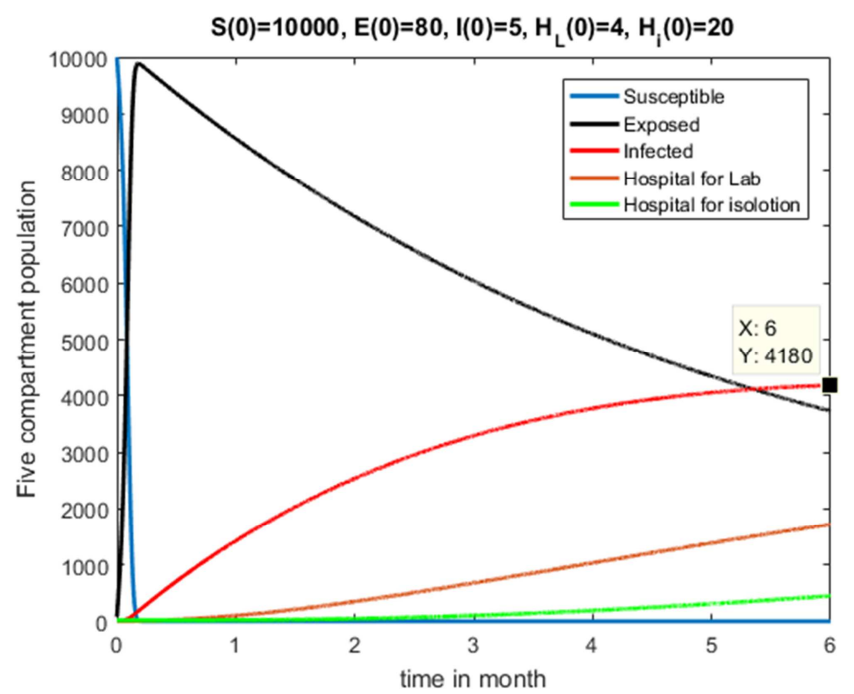

(a) No intervention is applied

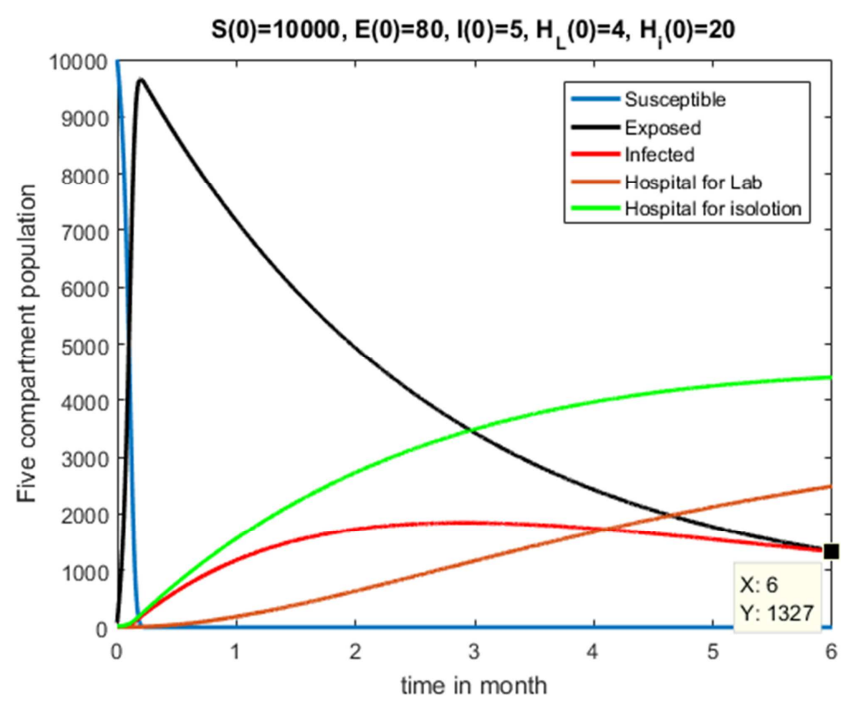

(b) Intervention is applied

Figure 3. The influence of intervention parameters $d, m_{1}, m_{2}$ on Reproductive number $R_{0}$.
Simulation study in Figure 3 (a), No intervention is applied, $\mathrm{f}=0, \mathrm{~m}_{1}=0, \mathrm{~m}_{2}=0$, shows that if the basic reproductive number $R_{0}$ is not less than unity $\left(R_{0}=4.14\right)$ then the infection will continue to spread in the population and also the susceptible population converges to zero as time diverges.

Simulations study in Figure 3 (b), Intervention is applied, $\mathrm{f}=0.20, \mathrm{~m}_{1}=0.20, \quad \mathrm{~m}_{2}=0.20$ shows that if the basic reproductive number $R_{0}$ is less than unity $\left(R_{0}=0.65\right.$.) then the spread of epidemic is under control and eventually stop.

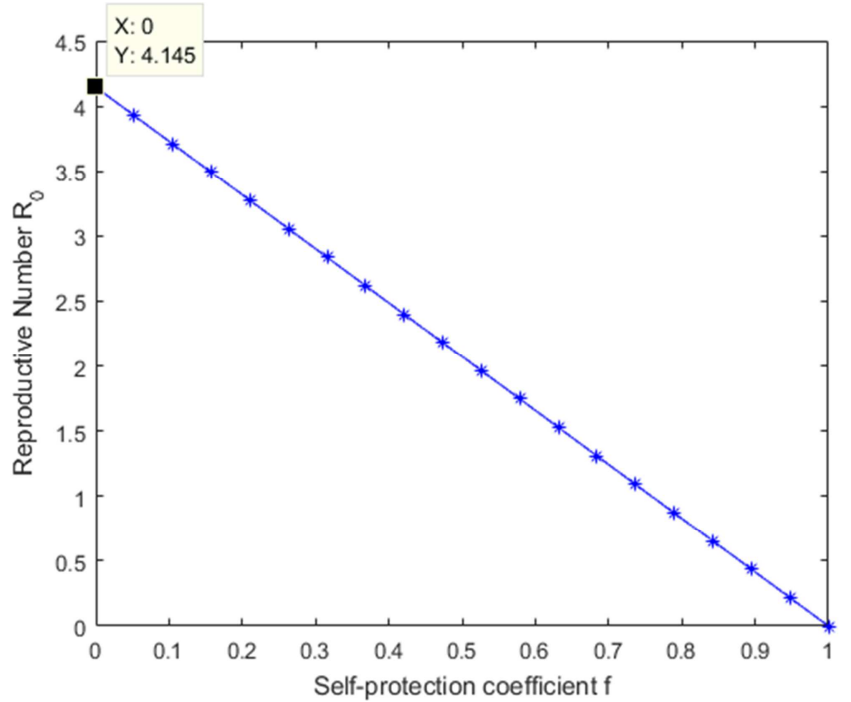

4(a). Relation between $\mathrm{f}$ and $\mathrm{R}_{0}$

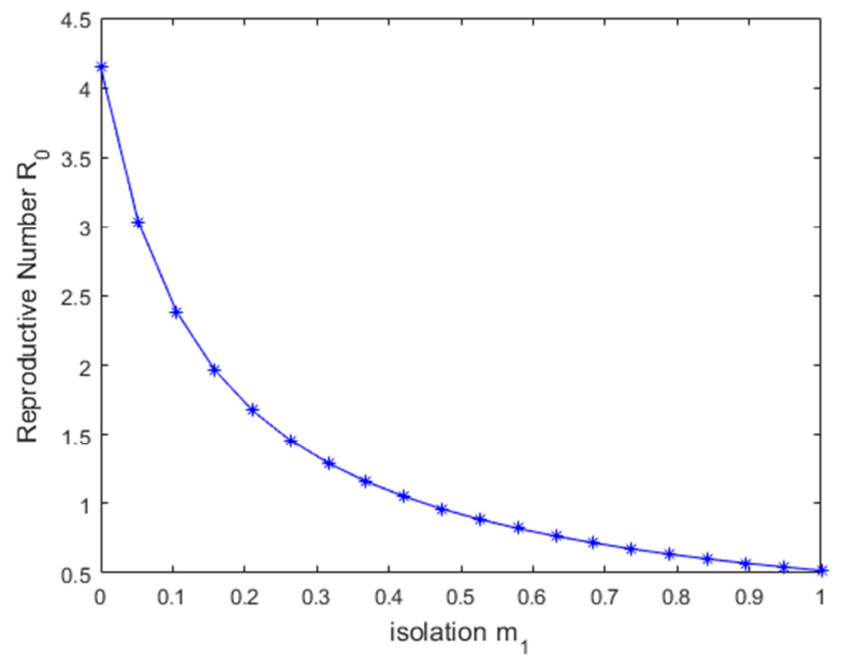

4(b). Relation between $\mathrm{m}_{1}$ and $\mathrm{R}_{0}$

Figure 4. The relationship between Intervention strategies ( $f$ and $\left.m_{1}\right)$ and Reproductive number $R_{0}$.

Finger 4(a), Show that the bigger value the self-protective coefficient, the smaller value of reproductive number $\mathrm{R}_{0}$ or smaller value the self-protective coefficient, the bigger value of reproductive number $\mathrm{R}_{0}$. Finger $4:(\mathrm{b})$, show that the relation between isolation from infected compartment and reproductive number $\mathrm{R}_{0}$ this graph show that isolating rate increases, the basic reproduction number $R_{0}$ will reduce. It tells us that quickly finding and isolating infected cases can 
effectively control the spread of epidemic.

\section{Conclusions}

In the present study we have formulated and analyzed a deterministic mathematical model for the dynamics of coronavirus (COVID-19) disease transmission. Self-protection coefficient and isolation are the best non-pharmaceutical controlling strategy for COVID-19 disease. Increasing the Self-protection coefficient and isolation rate will decrease in the rate of transmission of COVID-19 pandemic diseases. The reproduction number has been computed using next generation matrix method. We discussed the existence and stability of the disease free equilibrium points driven. The diseases free equilibrium points are shown locally asymptotically stable when $\mathrm{R}_{0}<1$. Also disease endemic equilibrium point of the model has been derived and it is globally asymptotically stable whenR $_{0}>1$. Simulation study and analysis of the model are performed by varying the social-distancing coefficient and isolation rate. It is observed that increasing of the Selfprotection coefficient and isolation rate on human has a significant impact on the rate of spread of COVID-19 transmission.

\section{References}

[1] Tweeten L., Barone E. and Wolfson E. "A Timeline of How the Wuhan Coronavirus Has Spread-And How the World Has Reacted". Time, 30 January 2020. Retrieved 08 February 2020 from https://time.com/5774366/how-coronavirus-spreadchina.

[2] World Health Organization. 2020a. "Novel Coronavirus (2019-nCoV) Situation Report - 10". World Health Organization, 30 January 2020. Retrieved 08 February 2020 from

https://www.who.int/docs/defaultsource/coronaviruse/situatio n-reports/20200130-sitrep10-ncov.pdf?sfvrsn=d0-b2e480_2.

[3] European Centre for Disease Prevention and Control. "Disease background of 2019nCoV". European Centre for Disease Prevention and Control. Retrieved 08 February 2020 from https://www.ecdc.europa.eu/en/2019-ncov-backgrounddisease. 2020 .

[4] Cui, J. et al. Origin and evolution of pathogenic coronaviruses. Nat. Rev. Microbiol. 17, 181-192 2019.

[5] Su, S. et al. Epidemiology, genetic recombination, and pathogenesis of coronaviruses. Trends Microbiol. 24, 490-502 (2016).

[6] Xia S, Liu Q, Wang Q, Sun ZW, Su S, Du LY, et al. Middle East respiratory syndrome coronavirus (MERS-CoV) entry inhibitors targeting spike protein. Virus research. 2014; 194: 200-210. doi: 10.1016/j.virusres.2014.10.007PMID:2545106.

[7] Memish Z A, Al-Tawfiq J A, Alhakeem R F, Assiri A, Alharby K D, Almahallawi M S, et al. Middle East respiratory syndrome coronavirus (MERS-CoV): A cluster analysis with implications for global management of suspected cases. Travel medicine and infectious disease. 2015; 13 (4): 311-314. doi: 10.1016/j.tmaid.2015.06.012 PMID: 26211569.
[8] Zhang RH. MERS-CoV: current research progress and prospect. Journal of Applied Virology. 2015; 4 (2): 30-36.

[9] Ferguson N M, Van Kerkhove M D. Identification of MERS$\mathrm{CoV}$ in dromedary camels. The Lancet infectious diseases. 2014; 14 (2): 93-94. doi: 10.1016/S1473-3099(13)70691-1 PMID: 24355867.

[10] Chowell G, Blumberg S, Simonsen L, Miller M A, Viboud C. Synthesizing data and models for the spread of MERS-CoV, 2013: Key role of index cases and hospital transmission. Epidemics. 2014; 9: 40-51. doi: 10.1016/j.epidem.2014.09.011 PMID: 25480133.

[11] Khan A, Farooqui A, Guan Y, Kelvin D J. Lessons to learn from MERS-CoV outbreak in South Korea. The Journal of Infection in Developing Countries. 2015; 9 (06): 543-546. doi: 10.3855/jidc.7278 PMID:26142661.

[12] Cauchemez S, Van Kerkhove MD, Riley S, Donnelly CA, Fraser C, Ferguso NM, et al. Transmission scenarios for Middle East Respiratory Syndrome Coronavirus (MERS-CoV) and how to tell them apart. Euro surveillance: bulletin Europeen sur les maladies transmissibles, European communicable disease bulletin. 2013; 18 (24).

[13] Lee SS, Wong NS. Probable transmission chains of Middle East respiratory syndrome coronavirus and the multiple generations of secondary infection in South Korea. International Journal of Infectious Diseases. 2015; 38: 65-67. doi: 10.1016/j.ijid.2015.07.014 PMID: 26216766.

[14] Sharif-Yakan A, Kanj S S. Emergence of MERS-CoV in the Middle East: origins, transmission, treatment, and perspectives. PLoS pathogens. 2014; 10 (12): e1004457. doi: 10.1371/journal.ppat.1004457 PMID: 25474536.

[15] Coronaviridae Study Group of the International Committee on Taxonomy of Viruses. The species Severe Acute Respiratory Syndrome-related coronavirus: classifying 2019-nCoV and naming it SARS-CoV-2. Nat Microbiol. 2020 Mar 2. [Epubahead of print].

[16] World Health Organization (WHO). Responding to community spread of COVID-19 interim guidance. 2020 Mar 7. $\quad$ Accessed $2020 \quad$ Mar 24. https://www.who.int/docs/defaultsource/coronaviruse/202003 07-responding-to-COVID-19-communitytransmissionfinal.pdf.

[17] Assiri, Abdullah; et al. "Epidemiological, demographic, and clinical characteristics of 47 cases of Middle East respiratory syndrome coronavirus disease from Saudi Arabia: a descriptive study". The Lancet Infectious Diseases. 13: 752761. doi: 10.1016/S1473-3099(13)70204-4. 9 September 2013.

[18] Assiri, A.; et al. "Hospital Outbreak of Middle East Respiratory Syndrome Coronavirus". NEJM. 369: 407-416. doi: 10.1056/NEJMoa1306742. Retrieved 20 May 2014.

[19] "Interim Guidance - Clinical management of severe acute respiratory infections when novel coronavirus is suspected: What to do and what not to do" (PDF). WHO. 2 November 2013. Retrieved 21 May 2014.

[20] The Health Protection Agency (HPA) UK Novel Coronavirus Investigation team "State of Knowledge and Data Gaps of Middle East Respiratory Syndrome Coronavirus (MERS-CoV) in Humans". Eurosurveillance. 14 March 2013. 
[21] WHO Coronavirus disease (COVID-19) technical guidance: Laboratory testing for 2019-nCoV in humans. https://www. who.int/emergencies/diseases/novel-coronavirus 2019/technic al-guidance/laboratory-guidance.

[22] "See Also". ProMED-mail. 20 September 2012. Retrieved 31 May 2013.

[23] Zhi-Qiang Xia, Juan Zhang, Ya-Kui Xue, Gui-Quan Sun, Zhen Jin Modeling the Transmission of Middle East Respirator Syndrome Corona Virus in the Republic of Korea. Article in PLoS ONE DOI: 10.1371/journal.pone.0144778.2015.

[24] Diekmann, O. and J. A. P. Heesterbeek. Mathematical Epidemiology of Infectious Diseases: Model Building, Analysis and Integration. Wiley: New York, NY. 2000.

[25] Heesterbeek H. et al. "Modeling infectious disease dynamics in the complex landscape of global health". Science, 347: aaa4339. 2015.

[26] Siettos C. I. and Russo L. "Mathematical modeling of infectious disease dynamics". Virulence, 4: 295-306.; 2013.

[27] Tuite A. R. and Fisman D. N. "Reporting, Epidemic Growth, and Reproduction Numbers for the 2019 Novel Coronavirus (2019-nCoV) Epidemic". Annals of Internal Medicine, 10.7326/M20-0358.; 2020.
[28] Lim P L. Middle East respiratory syndrome (MERS) in Asia: lessons gleaned from the South Korean outbreak. Transactions of the Royal Society of Tropical Medicine and Hygiene. 2015; 109 (9): 541-542. doi: 10.1093/trstmh/trv064 PMID: 26286944.

[29] Demsis Dejene, Purnachandra Rao Koya. Population Dynamics of Dogs Subjected To Rabies Disease. IOSR Journal of Mathematics IOSR Journal of Mathematics (IOSRJM) e-ISSN: 2278-5728, p-ISSN: 2319-765X. Volume 12, Issue 3 Ver. IV (May. - Jun. 2016), PP 110-120 www.iosrjournals.org DOI: 10.9790/5728-120304110120.

[30] LaSalle, J. P. "The Stability of Dynamical Systems". Regional Conference Series in Applied Mathematics, SIAM: Philadelphia, PA. 1976.

[31] The government of Wuhan homepage. Available from: http://english.wh.gov.cn/.

[32] J. A. Spencer, D. P. Shutt, S. K. Moser, H. Clegg, H. J. Wearing, H. Mukundan, et al., Epidemiological parameter review and comparative dynamics of influenza, respiratory syncytial virus, rhinovirus, human coronvirus, and adenovirus, medRxiv, 2020. 